\title{
Análise da adequação energética e proteica, de pacientes em terapia nutricional enteral internados em unidade de terapia intensiva em um hospital geral da Zona da Mata Mineira
}

\author{
Analysis of the energy and protein adequacy of patients undergoing enteral nutritional therapy ad- \\ mitted to an intensive care unit in a general hospital in Zona da Mata Mineira
}

DOI: $10.37111 /$ braspenj.2020351013

Fabiane Maciel Carneiro'

Mayla Cardoso Fernandes Toffolo ${ }^{2}$

\section{Unitermos:}

Terapia nutricional. Unidade de terapia intensiva. Recomendações nutricionais.

\section{Keywords:}

Nutrition therapy. Intensive care unit. Recommended dietary allowances.

\section{Endereço para correspondência: Mayla Cardoso Fernandes Toffolo Centro Universitário UNIFAMINAS}

Av Cristiano Ferreira Varella, 655 - Bairro Universitário - Muriae, MG, Brasil - 36880-000.

E-mail: maylaenut@gmail.com

\section{Submissão}

14 de novembro de 2019

Aceito para publicação

4 de fevereiro de 2020

\section{RESUMO}

Introdução: A terapia nutricional enteral é de grande importância na unidade de terapia intensiva (UTI), devido às condições clínicas desses pacientes, que, muitas vezes, apresentam inabilidade de alimentar-se por via oral. O objetivo deste estudo foi analisar as metas e adequação calórica e proteica calculada, prescrita e infundida nos pacientes internados na UTI de um hospital geral. Método: Trata-se de um estudo observacional, referente ao período de janeiro a junho de 2018. Foram incluídos pacientes com idade $\geq 19$ anos, que receberam nutrição enteral (NE) por pelo menos 72 horas. Foram analisadas as metas nutricionais energéticas e proteicas, calculadas, prescritas e infundidas nos pacientes internados. Resultados: 0 estudo obteve amostra de 94 pacientes. A adequação entre o calculado e prescrito foi $99,68 \%$, para caloria, e $91,18 \%$, para proteína. A razão entre prescrito e infundido foi de $96,38 \%$ e $95,97 \%$ para caloria e proteína, respectivamente. A relação entre calculado e infundido foi de 96,06 e $87,5 \%$ para caloria e proteína, respectivamente. Embora todos os valores médios dos indicadores avaliados estivessem maiores que $80 \%$, as médias de adequação entre as calorias prescrita vs. infundida e calculada vs. infundida e na análise das proteínas de todos os quesitos apresentaram $p<0,001$. Com relação aos indicadores de qualidade em terapia nutricional, observou-se que $26,6 \%$ da amostra obtiveram tempo de jejum pré-terapia nutricional $>48$ horas. A frequência de episódios de diarreia e constipação foi de $32,98 \%$ e $51,06 \%$, respectivamente. Um total de $78,72 \%$ dos pacientes atingiu suas necessidades nutricionais até o terceiro dia de NE. Conclusão: No presente trabalho, foi possível observar que a média calórica e proteica calculada, prescrita e infundida, por quilo de peso dos pacientes em TN, estiveram bem próximas ao mínimo recomendado. Entretanto, as médias foram diferentes, demonstrando a necessidade de melhor adequação de caloria e proteína calculada, prescrita e infundida.

\section{ABSTRACT}

Introduction: Enteral nutritional therapy is of great importance in the intensive care unit (ICU), due to the clinical conditions of these patients, who often have an inability to feed themselves orally. This study aims to analyze the goals and calculated caloric and protein adequacy prescribed and infused in hospitalized patients in the ICU of a general hospital. Methods: This is an observational and retrospective study, referring to the period from January to June 2018 . We included patients $\geq 19$ years old, who received enteral nutrition for at least 72 hours. It was analyzed the energy and protein nutritional goals, calculated, prescribed and infused in the inpatients. Values greater than $80 \%$ were adopted as a reference for adequacy. The paired t-test was performed in order to compare the calculated values of protein and calculated calorie, prescribed and infused. Statistical significance was set at $p<0.05$. Results: The study had a sample of 94 patients, with a mean age of $65.40 \pm 17.51$ years, being $54.26 \%$ male. The adequacy between calculated and prescribed nutrition was $99.68 \%$ for calorie and $91.18 \%$ for protein. The ratio between prescribed and infused nutrition was $96.38 \%$ and $95.97 \%$ for calorie and protein, respectively, and the relationship between calculated and infused was 96.06 and $87.5 \%$ for calorie and protein. Although all the means found obtained values greater than $80 \%$, the means between the calories, prescribed vs. infused, calculated vs. infused and protein analysis, in all the questions presented $p<0.001$. Regarding quality indicators in nutritional therapy (QINT), it was observed that $26.6 \%$ of the sample obtained fasting time of nutritional pre-therapy $>48$ hours. The frequency of diarrhea and constipation episodes were $32.98 \%$ and $51.06 \%$, respectively. A total of $78.72 \%$ of patients reached their nutritional needs by the third day of NE. Conclusion: In the present study, it was possible to observe that the calculated, prescribed and infused caloric and protein mean values per $\mathrm{kg}$ of patient weight in TN patients, were close to the recommended minimum. However, the means were different, demonstrating the need for better adequacy of calculated, prescribed and infused calories and protein.

1. Graduada em Nutrição pelo Centro Universitário UNIFAMINAS, Muriaé, MG, Brasil.

2. Professor Adjunto e Coordenador do curso de Nutrição do Centro Universitário UNIFAMINAS, Doutora em Saúde pelo Centro Universitário UNIFAMINAS, Muriaé, MG, Brasil. 


\section{INTRODUÇÃO}

Pacientes internados em unidade de terapia intensiva (UTI) geralmente exibem sinais de anorexia e inabilidade de alimentar-se por via oral durante dias ou meses, havendo, assim, recomendação de terapia nutricional enteral (TNE) e/ ou parenteral (TNP) ${ }^{1}$.

A TNE é de extrema relevância para os pacientes internados em UTI, pois em geral estes pacientes manifestam um estresse metabólico elevado, com intenso catabolismo proteico, progredindo com depleção muscular ${ }^{2}$. Recomendase início precoce da TNE, pelos consensos e diretrizes de sociedades nacionais e internacionais, para pacientes enfermos internados em estado grave, tendo em vista diminuir a deficiência energética, amenizar as alterações metabólicas presentes, catabolismo, redução de complicações infecciosas e diminuição do tempo de permanência na UTI'.

Caracteriza-se como nutrição precoce, a nutrição enteral iniciada em até 48 horas após admissão hospitalar, admissão UTI e/ou após abordagem cirúrgica. Este início pode alterar em virtude de características do paciente no ponto de vista clínico ou cirúrgico e da presença de estabilidade hemodinâmica, à medida que o paciente esteja com doses estáveis ou em desmame de substâncias vasoativas ${ }^{3}$.

As necessidades nutricionais variam de acordo com cada indivíduo, levando em consideração seu estado nutricional atual e habitual, idade, sexo, peso, estatura, atividade física, composição corporal e condições fisiológicas. A calorimetria indireta é indicada como o método seguro para a estimativa energética, porém na impossibilidade de utilização desse método, preconiza-se aplicar o gasto energético pelo cálculo de quilocaloria por quilo de peso corporal ${ }^{4}$. A Diretriz Brasileira de Terapia Nutricional no Paciente Grave projeto Diretrizes em Terapia Nutricional (DITEN) ${ }^{5}$ atualmente recomenda uma oferta energética menor nos três primeiros dias de $15-20 \mathrm{kcal} / \mathrm{kg} /$ dia, e, após o quarto dia de TNE, progredir para $25-30 \mathrm{kcal} / \mathrm{kg} /$ dia. A recomendação de oferta proteica é de 1,5 a 2,0 g/ $/ \mathrm{kg} / \mathrm{dia}$, não havendo distinção entre a fase que o paciente esteja ${ }^{5}$.

Atingir o suporte nutricional adequado pode não ser tão fácil, visto que ocorrem diversos fatores, os quais interferem na oferta nutricional programada, provocando a suspensão temporária e/ou permanente da dieta. Entre essas situações estão disfunção gastrointestinal (vômito, diarreia, distensão abdominal, resíduo gástrico aumentado), jejum para exames e procedimentos, retirada de sonda, obstrução da sonda e instabilidade clínica do paciente. Tais motivos favorecem que os indivíduos em TNE estejam propícios a receberem menor volume e aporte energético-proteico do que o prescrito ${ }^{6,7}$.

$\bigcirc$ déficit energético aumentado é um preditor importante em desfechos clínicos, visto que provavelmente não serão recompensados durante a internação na UTI ${ }^{8}$. Esses pacientes, quando sujeitos a ofertas nutricionais insuficientes, desenvolvem consequências clínicas, como aumento da mortalidade e da permanência no hospital e piora da qualidade de vida, representando um desafio à adequação da demanda energético-proteica desses pacientes ${ }^{6}$.

O acompanhamento diário da caloria e proteína calculado vs. prescrito vs. infundido é uma avaliação de identificação das causas da oferta inadequada da dieta enteral abaixo do previsto ${ }^{8}$. A supervisão da qualidade da terapia nutricional torna-se fundamental para identificar as não conformidades, em relação às metas de Indicadores de Qualidade em Terapia Nutricional (IQTN) ${ }^{9}$.

Os IQTN constituem o método mais aplicado para identificar as possíveis falhas do serviço prestado e providenciar soluções adequadas para melhorar a assistência ao paciente, visando à melhora contínua da qualidade ${ }^{10}$.

Diante do exposto, o presente trabalho visa analisar adequação calórica e proteica calculada, prescrita e infundida nos pacientes internados na UTI de um hospital geral da Zona da Mata Mineira.

\section{MÉTODO}

Trata-se de um estudo de caráter observacional e retrospectivo a partir do banco de dados existente na instituição, realizado na UTI geral de um hospital da Zona da Mata Mineira, referente ao período de janeiro a junho de 2018, após autorização pelo responsável da equipe multidisciplinar de terapia nutricional e aprovação pelo Comitê de Ética e Pesquisa da instituição, sob CAAE: 90916318.2 .0000 .5105$.

$\mathrm{O}$ estudo incluiu pacientes com idade maior ou igual a 19 anos, de ambos os sexos, que receberam NE exclusiva por pelo menos 72 horas, em topografia pré ou pós-pilórica, confirmado por exame de radiografia. Foram excluídos os pacientes em cuidados paliativos ou que receberam dieta parenteral e/ou oral simultaneamente.

As necessidades energéticas e proteicas foram calculadas a partir das recomendações sugeridas pela diretriz da Sociedade Brasileira de Nutrição Parenteral e Enteral (SBNPE) ${ }^{11}$, para pacientes graves, de acordo com o estado nutricional. Preconizou-se a recomendação de 20 a $25 \mathrm{kcal} / \mathrm{kg} / \mathrm{dia}$, na fase aguda, e 25 a $30 \mathrm{kcal} / \mathrm{kg} / \mathrm{dia}$, na fase anabólica, e 1,2 a 1,5 g ptn $/ \mathrm{kg} / \mathrm{dia}$, para catabolismo moderado, e 1,5 a 2,0 $\mathrm{g}$ ptn $/ \mathrm{kg} /$ dia, em pacientes hipercatabólicos. O peso utilizado para o cálculo das necessidades nutricionais foi o registrado em prontuário da última pesagem ou o estimado por meio de equações preditivas para adultos e idosos.

Foram analisadas as metas nutricionais energéticas e proteicas, calculadas, prescritas e o infundido nos pacientes internados. Foram utilizados, como referencial de adequação, valores superiores a $80 \%$. As análises foram realizadas por meio das seguintes fórmulas:

- Adequação caloria/proteína calculado vs. prescrito (\%) = caloria/proteína calculado $\times 100$ / caloria/proteína prescrito; 
- Adequação da caloria/proteína prescrito vs. infundido $(\%)=$ caloria/proteína prescrito $\times 100$ / caloria/proteína infundido;

- Adequação caloria/proteína calculado vs. infundido (\%) = caloria/proteína calculado $\times 100$ / caloria/proteína infundido.

Os valores energéticos e proteicos, prescritos e infundido foram calculados pela média da caloria e proteína diária calculada, prescrita e infundido de NE, respectivamente, coletados nos prontuários a partir do quarto dia de TNE até óbito, alta na unidade ou início de outra via complementar ou exclusiva.

A escolha de analisar a oferta da NE após o quarto dia foi estabelecida segundo Waitzberg et al. ${ }^{12}$, como sendo o período necessário para atingir o aporte energético máximo do paciente.

Os indicadores de qualidades da TNE aplicados foram: tempo de jejum antes da TNE, frequência de diarreia e constipação e pacientes com TNE que atingiram 100\% das necessidades nutricionais em até três dias (Quadro 1) 13.

Os dados foram analisados no programa estatístico Statistical Package for the Social Sciences (SPSS), versão 23.0 e apresentados em média e desvio padrão para as variáveis quantitativas (idade, tempo de TNE, metas nutricionais calculadas, prescritas e valor infundido), e frequência absoluta e relativa para as categóricas (sexo, diagnóstico de internação, desfecho do paciente, indicadores de qualidade e adequações).

Foi realizado teste de normalidade Kolmogorov Smirnov para atestar a normalidade dos dados. O teste t pareado foi

\begin{tabular}{|c|c|c|}
\hline Indicador & Fórmulas & Metas \\
\hline $\begin{array}{l}\text { Pacientes com } \\
\text { tempo de jejum } \\
\text { inadequado } \\
\text { pré-terapia } \\
\text { nutricional } \\
\text { (> } 48 \text { horas) }\end{array}$ & $\begin{array}{c}N^{\circ} \text { de pacientes candidatos à } \\
\text { TNE com jejum }>48 \text { horas x } 100 \\
N^{\circ} \text { de pacientes candidatos à } \\
\text { TNE }\end{array}$ & $<20 \%$ \\
\hline $\begin{array}{l}\text { Frequência de } \\
\text { episódios de } \\
\text { diarreia em } \\
\text { pacientes em TNE }\end{array}$ & $\begin{array}{c}\mathrm{N}^{\circ} \text { de pacientes em TNE com } \\
\text { diarreia } \times 100 \\
\mathrm{~N}^{\circ} \text { de pacientes em TNE }\end{array}$ & $\leq 10 \%$ \\
\hline $\begin{array}{l}\text { Frequência de } \\
\text { constipação } \\
\text { intestinal em } \\
\text { pacientes em TNE }\end{array}$ & $\begin{array}{c}\mathrm{N}^{\circ} \text { de pacientes em TNE com } \\
\text { constipação x } 100 \\
\mathrm{~N}^{\circ} \text { de pacientes em TNE }\end{array}$ & $<20 \%$ \\
\hline $\begin{array}{l}\text { Taxa de pacientes } \\
\text { com TNE que } \\
\text { atingiram } 100 \% \\
\text { das necessidades } \\
\text { nutricionais em } \\
\text { até } 3 \text { dias }\end{array}$ & $\begin{array}{c}\mathrm{N}^{\circ} \text { de pacientes em TNE } \\
\mathrm{N}^{\circ} \text { de pacientes TNE que } \\
\text { atingiram } 100 \% \text { de adequação } \\
\text { das necessidades calórico e/ou } \\
\text { proteica } \times 100 \\
\mathrm{~N}^{\circ} \text { total de pacientes com TNE }\end{array}$ & $\geq 80 \%$ \\
\hline
\end{tabular}

Fonte: Gandolfo et al.13. utilizado para comparar os valores entre proteína e calorias calculada, prescrita e infundida. Assumiu-se com nível de significância estatística o valor $p<0,05$.

\section{RESULTADOS}

O estudo contou com uma amostra de 94 pacientes, com média de idade de 65,40 $\pm 17,51$ anos, sendo $54,26 \%$ do sexo masculino. Os principais diagnósticos de internação foram acidente vascular encefálico (AVE) $(19,15 \%)$, rebaixamento do nível de consciência $(11,70 \%)$ e pneumonia $(9,58 \%)$. O número médio de dias que os pacientes permaneceram em TNE foi de 18,49 $\pm 14,94$ dias. Para o grupo avaliado, houve desfecho de $50 \%$ de óbito (Tabela 1).

Tabela 1 - Características demográficas, clínicas e sobre a TNE em pacientes internados em UTI de um hospital da Zona da Mata Mineira, 2018.

\begin{tabular}{lc}
\hline Características & Resultados \\
\hline Idade (anos) & $65,40 \pm 17,51^{*}$ \\
\hline Sexo & \\
Masculino $(n=51)$ & $54,26 \%$ \\
Feminino $(n=43)$ & $45,74 \%$ \\
\hline
\end{tabular}

\section{Diagnóstico}

Acidente vascular encefálico $(n=18)$

$19,15 \%$

Rebaixamento do nível de consciência $(n=11)$

$11,70 \%$

Pneumonia $(\mathrm{n}=9)$

$9,58 \%$

Doença pulmonar obstrutiva crônica $(n=8)$

$8,51 \%$

Traumatismos cranioencefálico $(n=7)$

$7,45 \%$

Cirurgia abdominal $(n=4)$

$4,25 \%$

Politrauma $(n=4)$

$4,25 \%$

Edema agudo de pulmão $(n=2)$

$2,13 \%$

Insuficiência cardíaca crônica $(n=2)$

$2,13 \%$

Alzheimer $(\mathrm{n}=2)$

$2,13 \%$

Pancreatite $(n=2)$

$2,13 \%$

Anorexia $(\mathrm{n}=2)$

$2,13 \%$

Convulsão $(n=2)$

$2,13 \%$

Sepse $(n=1)$

$1,06 \%$

Neoplasia $(n=1)$

$1,06 \%$

Cirrose $(n=1)$

$1,06 \%$

Outros $(n=18)$

$19,15 \%$

Tempo em TNE (dias)

$18,49 \pm 14,94^{*}$

\section{Desfecho do Paciente}

Alta da UTI $(n=40)$

$42,55 \%$

Óbito $(n=47)$

$50,00 \%$

Outros $^{\star \star}(\mathrm{n}=7)$

$7,45 \%$

*Média e desvio padrão; **Pacientes transferidos para outro hospital ou que permaneceram na UTI até a data limite da pesquisa.

$\mathrm{TNE}$ = terapia nutricional enteral; UTI = unidade de terapia intensiva. 
As metas calóricas e proteicas calculadas, prescritas e infundidas encontram-se descritas na Tabela 2 . A adequação entre o calculado e prescrito foi de $99,68 \%$ para caloria e $91,18 \%$ para proteína. A razão entre prescrito e infundido foi de $96,38 \%$ e $95,97 \%$ para caloria e proteína, respectivamente. A relação entre calculado e infundido foi de $96,06 \%$ para caloria e $87,50 \%$ para proteína.

Embora os indicadores tenham atingido as metas propostas, os valores médios foram diferentes entre si, sendo semelhantes apenas para adequação energética calculada vs. prescrita $(p>0,005)$.

Com relação aos IQTN, observou-se que $26,6 \%$ da amostra obtiveram tempo de jejum pré-terapia nutricional $>48$ horas. A frequência de episódios de diarreia e constipação foram de $32,98 \%$ e $51,06 \%$, respectivamente. Um total de $78,72 \%$ dos pacientes atingiu suas necessidades nutricionais até o terceiro dia de NE. Todos os resultados observados estiveram em desacordo com as metas propostas (Tabela 3).

Tabela 2 - Média e adequação (\%) das metas nutricionais calculada, prescrita e valor infundido em pacientes internados na UTI de um hospital da Zona da Mata Mineira, 2018.

\begin{tabular}{|c|c|}
\hline Variáveis & Valor Observado \\
\hline \multicolumn{2}{|l|}{ Meta Calculada } \\
\hline Energia (kcal/kg/dia) & $21,59 \pm 4,86^{*}$ \\
\hline Proteína (g/kg/dia) & $1,36 \pm 0,15^{\star}$ \\
\hline \multicolumn{2}{|l|}{ Meta Prescrita } \\
\hline Energia (kcal/kg/dia) & $21,52 \pm 4,88^{*}$ \\
\hline Proteína (g/kg/dia) & $1,24 \pm 0,20^{*}$ \\
\hline \multicolumn{2}{|l|}{ Valor Infundido } \\
\hline Energia (kcal/kg/dia) & $20,74 \pm 5,09^{*}$ \\
\hline Proteína (g/kg/dia) & $1,19 \pm 0,22^{*}$ \\
\hline \multicolumn{2}{|l|}{ Adequação (\%) } \\
\hline \multicolumn{2}{|l|}{ Calculado x Prescrito } \\
\hline Energia & $99,68 \%$ \\
\hline$p$-valor** & 0,743 \\
\hline Proteína & $91,18 \%$ \\
\hline$p$-valor** & 0,000 \\
\hline \multicolumn{2}{|l|}{ Prescrito x Infundido } \\
\hline Energia & $96,38 \%$ \\
\hline p-valor ${ }^{\star \star}$ & 0,001 \\
\hline Proteína & $95,97 \%$ \\
\hline$p$-valor** & 0,000 \\
\hline \multicolumn{2}{|l|}{ Calculado x Infundido } \\
\hline Energia & $96,06 \%$ \\
\hline$p$-valor** & 0,021 \\
\hline Proteína & $87,50 \%$ \\
\hline$p$-valor ${ }^{\star *}$ & 0,000 \\
\hline
\end{tabular}

* Média e desvio padrão; **Teste t pareado.

UTI = Unidade de terapia intensiva.
Tabela 3 - Resultados dos indicadores de qualidades em TNE da UTI de um hospital da Zona da Mata Mineira, 2018.

\begin{tabular}{lcc}
\hline $\begin{array}{l}\text { Indicador de } \\
\text { Qualidade }\end{array}$ & $\begin{array}{c}\text { Valor } \\
\text { encontrado }\end{array}$ & $\begin{array}{c}\text { Meta } \\
\text { proposta }\end{array}$ \\
\hline $\begin{array}{l}\text { Pacientes com tempo de jejum inadequado } \\
\text { pré-terapia nutricional (> 48 horas) }\end{array}$ & $26,60 \%$ & $<20 \%$ \\
$\begin{array}{l}\text { Frequência de episódios de diarreia em } \\
\text { pacientes em TNE }\end{array}$ & $32,98 \%$ & $\leq 10 \%$ \\
$\begin{array}{l}\text { Frequência de constipação intestinal em } \\
\text { pacientes em TNE }\end{array}$ & $51,06 \%$ & $<20 \%$ \\
$\begin{array}{l}\text { Taxa de pacientes com TNE que atingiram } \\
100 \% \text { das necessidades nutricionais em } \\
\text { até 3 dias }\end{array}$ & $78,72 \%$ & $\geq 80 \%$ \\
\hline
\end{tabular}

TNE = terapia nutricional enteral; UTI = unidade de terapia intensiva.

\section{DISCUSSÃO}

Conforme a diretriz atual, deve-se atingir a necessidade plena do paciente no quarto dia pós início da TNE, pois nos primeiros dias os doentes graves possuem uma produção endógena de energia, caracterizada pela fase de injúria aguda. Os estudos citados como referência na diretriz demonstraram que os pacientes com aporte menor de $70 \%$ das necessidades calóricas, nas primeiras 72 horas, obtiveram melhor desfecho em termos de sobrevida ${ }^{5}$.

Porém, como a condução e elaboração do estudo ocorreu no primeiro semestre de 2018, utilizou-se para base de cálculo e avaliação dos resultados a referência da SBNPE ${ }^{11}$.

No presente trabalho, a maioria dos pacientes estudados $(78,72 \%)$ atingiu suas necessidades energéticas/proteicas totais até o terceiro dia de $\mathrm{NE}$, estando próximo à meta estabelecida por Gandolfo et al. ${ }^{13}$, que padronizaram valores $\geq 80 \%$.

Tal meta pode não ter sido alcançada pois se trata de uma UTI geral e considera-se que os pacientes graves podem apresentar uma progressão dificultada por complicações clínicas. que dificultam a aporte adequado e os benefícios dos nutrientes, entre as complicações estão acidose, hiperglicemia, hipernatremia, instabilidade hemodinâmica e uso de aminas vasoativas ${ }^{14}$.

No presente estudo, os valores encontrados da média calórica calculada, prescrita e infundida estão de acordo com o preconizado pela diretriz da SBNPE ${ }^{11}$, para pacientes graves, as médias calóricas encontradas estão de acordo com o recomendado na fase aguda que estabelece $20-25 \mathrm{kcal} /$ $\mathrm{kg} /$ dia. No estudo conduzido por De Jonghe et al. ${ }^{15}$, com 51 pacientes de UTI em TNE, foi alcançada uma média de necessidade energética de $28,1 \pm 4,7 \mathrm{kcal} / \mathrm{kg} / \mathrm{dia}$, sendo que $\circ$ prescrito foi de $22 \pm 8,6 \mathrm{kcal} / \mathrm{kg} /$ dia e infundida de $20,0 \pm 7,9 \mathrm{kcal} / \mathrm{kg} /$ dia. A média calórica prescrita e infundida foi bem próxima nos dois estudos, demonstrando adequação com o recomendado pela SBNPE ${ }^{11}$. 
As recomendações de calorias e proteínas publicadas pelo DITEN ${ }^{5}$ diferem da referência utilizada no trabalho para o cálculo das necessidades calorias, pois sugere o uso de 15 a $20 \mathrm{kcal} / \mathrm{kg} / \mathrm{dia}$, nos primeiros três dias, ou seja, na fase aguda, estando a fase anabólica mantida a mesma recomendação e as necessidades proteicas 1,5 a $2,0 \mathrm{~g} / \mathrm{kg} /$ dia, sem diferença entre as fases ${ }^{5}$.

O percentual de adequação obtido para caloria calculada vs. prescrita foi de $99,68 \%$, prescrita vs. infundida $96,38 \%$ e calculada vs. infundido $96,06 \%$, assim, todos os parâmetros avaliados estão em acordo com a meta proposta de $>80 \%$.

Porém observaram-se médias energéticas diferentes para prescrito vs. infundido e calculado vs. infundido $(p<0,05)$, indicando a necessidade de melhorar o valor calórico infundido, visando aproximar mais do calculado.

Um estudo realizado por Biolo et al. ${ }^{16}$ observou que tanto a hiperalimentação quanto a restrição calórica e proteica se associam à diminuição da efetividade anabólica e à degeneração muscular esquelética. Em caso de superoferta, observa-se aumento da atividade inflamatória e do estresse oxidativo, associado a metabolismo muscular esquelético e inflamação sistêmica. Na pesquisa supracitada, identificouse que, em situações de baixa oferta calórica e proteica, o trabalho muscular desempenhou um papel importante na proteção contra atrofia e diminuição dos processos inflamatórios, destacando a necessidade de uma faixa ótima de oferta energética e proteica ${ }^{16}$.

As médias proteicas obtida no atual estudo estão de acordo com o recomendado pela diretriz da SBNPE ${ }^{11}$, para pacientes graves na fase aguda, que indica o uso de 1,2 a $1,5 \mathrm{~g} / \mathrm{kg} /$ dia. Ao se comparar esses valores com o estudo realizado com 53 pacientes de UTI em NE, realizado em 2015 no hospital universitário de São Luís - MA, verifica-se valores de 1,27, 0,95, 0,83 g/ $\mathrm{kg} /$ dia de proteína calculado, prescrita e administrada respectivamente, estando abaixo do encontrado em nosso estudo e em desacordo com a meta estabelecida ${ }^{17}$.

A proteína é o principal macronutriente para os pacientes críticos, sendo responsável pelo aumento da função imunológica, manutenção da massa magra e cicatrização das feridas. Segundo os especialistas, esses pacientes possuem demanda aumentada de proteínas e a oferta adequada está relacionada a desfechos positivos?.

O percentual de adequação para proteína do presente estudo em todos os quesitos foi superior a $87 \%$, estando em acordo com a meta proposta. Contudo, também observou médias diferentes $(p<0,001)$ em todos os itens (proteína calculada, prescrita e infundida), tal resultado sinaliza que se deve buscar uma melhor prescrição e/ou infusão da dieta, visando aproximar a oferta e adequação à necessidade do paciente.
Destaca-se, no atual estudo, um bom percentual de adequação entre os valores de energia e proteína infundida e prescrita, estando acima de $95 \%$. Um estudo na UTI do Hospital Universitário da Universidade de São Paulo, no segundo semestre dos anos de 2008 e 2009, encontrou uma adequação inferior, de $88,2 \%{ }^{18}$.

A média de permanência em TNE foi de 18,49 \pm 14,94 dias, acima do valor encontrado por Teixeira et al. ${ }^{2}$, em um estudo observacional realizado com 53 pacientes da UTI do hospital universitário da USP, que verificaram média de 12,8 $\pm 11,2$ dias. Segundo o Sistema de Informação Hospitalar do Sistema Único de Saúde, o tempo médio de permanência em UTI é de 7,6 dias, bem inferior à média encontrada no presente estudo 19 .

Um estudo realizado por Gomes et al. ${ }^{17}$ encontrou $54,72 \%$ de mortalidade entre a amostra analisada, valor próximo ao presente estudo, onde observou-se $50 \%$ de óbitos entre os pacientes. Os dois estudos apresentaram ocorrência de óbitos acima do valor de referência para pacientes de UTI presentes na literatura, que se encontra entre $24 \%$ e $47 \%{ }^{19}$.

Dentre os pacientes avaliados, $26,6 \%$ tiveram tempo de jejum $>48$ horas, caracterizando não conformidade com a meta de indicador proposto por Gandolfo et al. ${ }^{13}$. A introdução precoce da NE em UTI, quando iniciada nas primeiras 48 horas de admissão hospitalar, está relacionada com menor incidência de infecções, de resposta catabólica e manutenção da integridade intestinal, reduzindo o tempo de permanência hospitalar ${ }^{20}$. $\bigcirc$ jejum prolongado está associado a complicações, como atrofia da mucosa intestinal, que favorece o aumento da permeabilidade e translocação bacteriana da luz para os linfonodos mesentéricos, podendo conduzir a sepse e óbito ${ }^{21}$.

Comparado com estudo de Pasinato et al. ${ }^{22}$, realizado com 115 pacientes da UTI do Hospital de Clínicas de Porto Alegre, no qual $63 \%$ dos pacientes críticos receberam NE precoce, no presente estudo, a maioria dos pacientes $(73,4 \%)$ também iniciou a NE em até 48 horas.

Observou-se, no presente estudo, um alto percentual de pacientes em TNE que apresentaram diarreia e constipação intestinal, estando em desacordo com as metas propostas por Gandolfo et al. ${ }^{13}$.

A constipação intestinal é bastante comum em pacientes que estão utilizando nutrição enteral, relacionados ao uso de medicamentos que retardam a motilidade do trato gastrointestinal, entre eles aparece os benzodiazepínicos e os opioides e a desidratação como fator complicador ${ }^{23}$.

De acordo com Borges et al. ${ }^{24}$, a grande ocorrência de diarreia está relacionada à alta frequência de prescrições de múltiplos antibióticos e à antibioticoterapia de longa duração. Os autores observaram que a antibioticoterapia resultou em aumento de $16 \%$ no risco de diarreia, e o acréscimo de 
outro antibiótico à terapia indica um aumento de $65 \%$ nos episódios de diarreia.

Segundo Cremonini et al. ${ }^{25}$, a ocorrência de diarreia em pacientes com NE varia de acordo com o conceito aplicado para determinação de diarreia, estando citados nas literaturas valores de $16 \%$ a $63 \%$.

O presente estudo observou uma incidência de 32,98\% de diarreia nos pacientes em uso de NE. Comparado com o estudo realizado na UTI do hospital universitário da USP, em 2005 e 2006, que encontrou 36\% e 17\%, respectivamente, contudo, o valor encontrado no estudo aproxima-se do comparado. A redução de episódios de diarreia na UTI é marcada como aspecto positivo, apesar de não ser motivo de interrupção na administração da dieta ${ }^{14}$.

A frequência de constipação intestinal no atual estudo foi de $51,06 \%$. Os resultados observados em outros estudos variam de $15 \%$ a $83 \%$, podendo estar associados às diferentes definições de constipação entre os estudos. Um estudo de revisão listou os principais manejos, a fim de minimizar a ocorrência de constipação, entre elas são citados: protocolos de identificação, quantificação e tratamento da constipação; diagnóstico de constipação em pacientes graves na frequência de eliminação fecal, quantidade e aparência pela escala de Bristol ${ }^{26}$.

A equipe da nutrição deve estar atenta ao início e progressão da dieta e, quando possível, contemplar dietas com fibras e atentar a condições de propiciem o surgimento de constipação, como imobilização prolongada, uso de opioides, sedação excessiva, bloqueadores neuromusculares, desidratação, distúrbios eletrolíticos, tentando sempre minimizar a exposição a estas condições ${ }^{26}$.

A constipação intestinal é uma complicação habitualmente reconhecida em pacientes graves e pode estar ligada ao prognóstico desses pacientes, devendo ser diagnosticada e tratada ${ }^{26}$.

O presente estudo contribuiu para uma visão geral do serviço de nutrição presente, destacando os pontos que podem ser melhorados, como melhor média da prescrição calórica e proteica e procurar melhorar a assistência nutricional na TNE, de acordo com as metas propostas pelos indicadores de qualidade.

\section{CONCLUSÃO}

No presente trabalho, foi possível observar que a média calórica e proteica calculada, prescrita e infundida, por $\mathrm{kg}$ de peso dos pacientes em TN, esteve acima do mínimo recomendado pela SBNPE, sendo estes valores adequados.

Embora a adequação calórica e proteica calculada, prescrito e infundida tenha sido superior a $80 \%$ conforme preconizado, as médias foram diferentes. Faz-se necessário um melhor acompanhamento da prescrição e infusão da dieta, visando à aproximação das necessidades nutricionais, destes pacientes estabelecidas pelo nutricionista e equipe multidisciplinar.

Conclui-se, no presente estudo, que houve inadequação nos resultados dos IQTN analisados, sugere-se à equipe multidisciplinar a criação e a implantação de protocolos específicos, a fim de diminuir o tempo de jejum pré TNE; monitorar a disfunção do trato gastrointestinal desses pacientes; e estabelecer uma monitorização rotineira do aporte nutricional, disposto a assegurar a conformidade plena. Além disso, destaca-se a necessidade de continuar a pesquisa, monitorizando constantemente a qualidade da TNE, a fim de assegurar melhorias contínuas no serviço.

\section{REFERÊNCIAS}

1. Borges VC, Barone MG, Oliveira PM. Terapia nutricional enteral precoce. In: Toledo $\mathrm{D}$, Castro $\mathrm{M}$, eds. Terapia nutricional em UTI. Rio de Janeiro: Rubio; 2015. p.91-6.

2. Teixeira ACC, Caruso L, Soriano FG. Terapia nutricional enteral em unidade de terapia intensiva: infusão versus necessidades. Rev Bras Ter Intensiva. 2006;18(4):331-7.

3. McClave SA, Martindale RG, Vanek VW, McCarthy M, Roberts P, Taylor B, et al. A.S.P.E.N. Board of Directors; American College of Critical Care Medicine; Society of Critical Care Medicine. Guidelines for the provision and assessment of nutrition support therapy in the adult critically ill patient: Society of Critical Care Medicine (SCCM) and American Society for Parenteral and Enteral Nutrition (A.S.P.E.N.). JPEN J Parenter Enteral Nutr. 2009;33(3):277-316.

4. Sociedade Brasileira de Nutrição Parenteral e Enteral, Sociedade Brasileira de Clínica Médica, Associação Brasileira de Nutrologia. Recomendações nutricionais para adultos em terapia nutricional enteral e parenteral. Projeto Diretrizes. São Paulo: Associação Médica Brasileira e Conselho Federal de Medicina; 2011.

5. Sociedade Brasileira de Nutrição Parenteral e Enteral. Diretrizes brasileira de terapia nutricional. Projeto DITEN. BRASPEN J. 2018;33(Supl 1):1-46.

6. Schlaad JRM, Shiroma GM. Como monitorar a adequação da terapia nutricional. In: Toledo D, Castro M, eds. Terapia nutricional em UTI. Rio de Janeiro: Rubio; 2015. p.369-72.

7. Oliveiro-Filho RS, Tamburrino AC, Trevisani VS, Rosa VM. Main barriers in control of energy-protein deficit in critical oncologic patient at nutritional risk. J Integr Oncol. 2016;5(1):156. doi:10.4172/2329-6771.1000156

8. Santana NMA, Vieira LL, Dias DAM, Braga CC, Costa RM. Inadequação calórica e proteica e fatores associados em pacientes graves. Rev Nutr. 2016;29(5):645-54.

9. McClave AS, Taylor BE, Martindale RG, Warren MM, Johnson DR, Braunschweig C, Society of Critical Care Medicine; American Society for Parenteral and Enteral Nutrition. Guidelines for the provision and assessment of nutrition support therapy in the adult critically ill patient: Society of Critical Care Medicine (SCCM) and American Society for Parenteral and Enteral Nutrition (A.S.P.E.N.). JPEN J Parenter Enteral Nutr. 2016;40(2):159-211.

10. Verotti CCG, Ceniccola GD. Indicadores de qualidade em terapia nutricional na unidade de terapia intensiva. In: Toledo D, Castro M, eds. Terapia nutricional em UTI. Rio de Janeiro: Rubio; 2015. p.361-7. 
11. Sociedade Brasileira de Nutrição Parenteral e Enteral. Terapia nutricional no paciente grave. Projeto Diretrizes. São Paulo: Associação Médica Brasileira e Conselho Federal de Medicina; 2011.

12. Waitzberg DL, Fadul RA, van Aanholt DPJ. Indicação e técnicas de ministração em nutrição enteral. In: Waitzberg DL, ed. Nutrição oral, enteral e parenteral na prática clínica. $3^{\mathrm{a}}$ ed. São Paulo: Atheneu; 2006. p.561-71.

13. Gandolfo AS. Indicadores de nutrição clínica. In: Isosaki M, Gandolfo AS, Jorge AL, Evazian D, Castanheira OJNV, Aparecida e Bittar F, eds. Indicadores de nutrição hospitalar. São Paulo: Editora Atheneu; 2015. p.50-6.

14. Aranjues AL, Teixeira ACC, Caruso L, Soriano FG. Monitoração da terapia nutricional enteral em UTI: indicador de qualidade? O mundo da saúde São Paulo. 2008;32(1):16-23.

15. De Jonghe B, Appere-De-Vechi C, Fournier M, Tran B, Merrer J, Melchior JC, et al. A prospective survey of nutritional support practices in intensive care unit patients: what is prescribed? What is delivered? Crit Care Med. 2001;29(1):8-12.

16. Biolo G, Fleming RY, Maggi SP, Nguyen TT, Herndon DN, Wolfe RR. Inverse regulation of protein turnover and amino acid transport in skeletal muscle of hypercatabolic patients. J Clin Endocrinol Metab. 2002;87(7):3378-84.

17. Gomes RS, Cabral NAL, Oliveira ATV. Qualidade da terapia nutricional enteral em unidades de terapia intensiva. BRASPEN J. 2017;32(2):165-9.

18. Oliveira NS, Caruso L, Bergamaschi DP, Cartolano FC, Soriano FG. Impacto da adequação da oferta energética sobre a mortalidade em pacientes de UTI recebendo nutrição enteral. Rev Bras Ter Intensiva. 2011;23(2):183-9.

19. Nunes AP, Zanchim MC, Kümpel DA, Rodrigues TP, Zanin J. Adequação calórico-proteica da terapia nutricional enteral em pacientes críticos de um hospital de alta complexidade do Rio Grande do Sul. BRASPEN J. 2018;33(2):116-21.

20. Campanella LCA, Silveira BM, Rosário Neto O, Silva AA. Terapia nutricional enteral: a dieta prescrita é realmente infundida? Rev Bras Nutr Clín. 2008;23(1):21-5.

21. Côrtes JFF, Fernandes SL, Nogueira-Maduro IPN, Basile Filho A, Suen VMM, Santos JE, et al. Terapia nutricional no paciente criticamente enfermo. Medicina. 2003;36(2/4):394-8.

22. Pasinato VF, Berbigier MC, Rubin BA, Castro K, Moraes RB, Perry IDS. Terapia nutricional enteral em pacientes sépticos na unidade de terapia intensiva: adequação às diretrizes nutricionais para pacientes críticos. Rev Bras Ter Intensiva. 2013;25(1):17-24.

23. Bittencourt AF. Diarreia e constipação intestinal em terapia nutricional enteral [Dissertação de Mestrado]. São Paulo: Faculdade de Medicina da Universidade de São Paulo; 2013.

24. Borges SL, Pinheiro BV, Pace FHL, Chebli JMF. Diarreia nosocomial em unidade de terapia intensiva: incidência e fatores de risco. Arq Gastroenterol. 2008;45(2):117-23.

25. Cremonini F, Di Caro S, Nista EC, Bartolozzi F, Capelli G, Gasbarrini G, et al. Meta-analysis: the effect of probiotic administration on antibiotic-associated diarrhoea. Aliment Pharmacol Ther. 2002;16(8):1461-7.

26. Azevedo RP, Freitas FGR, Ferreira EM, Machado FR. Constipação intestinal em terapia intensiva. Rev Bras Ter Intensiva. 2009;21(3):324-31.

Local de realização do estudo: Centro Universitário UNIFAMINAS, Muriaé, MG, Brasil.

Conflito de interesse: Os autores declaram não haver. 